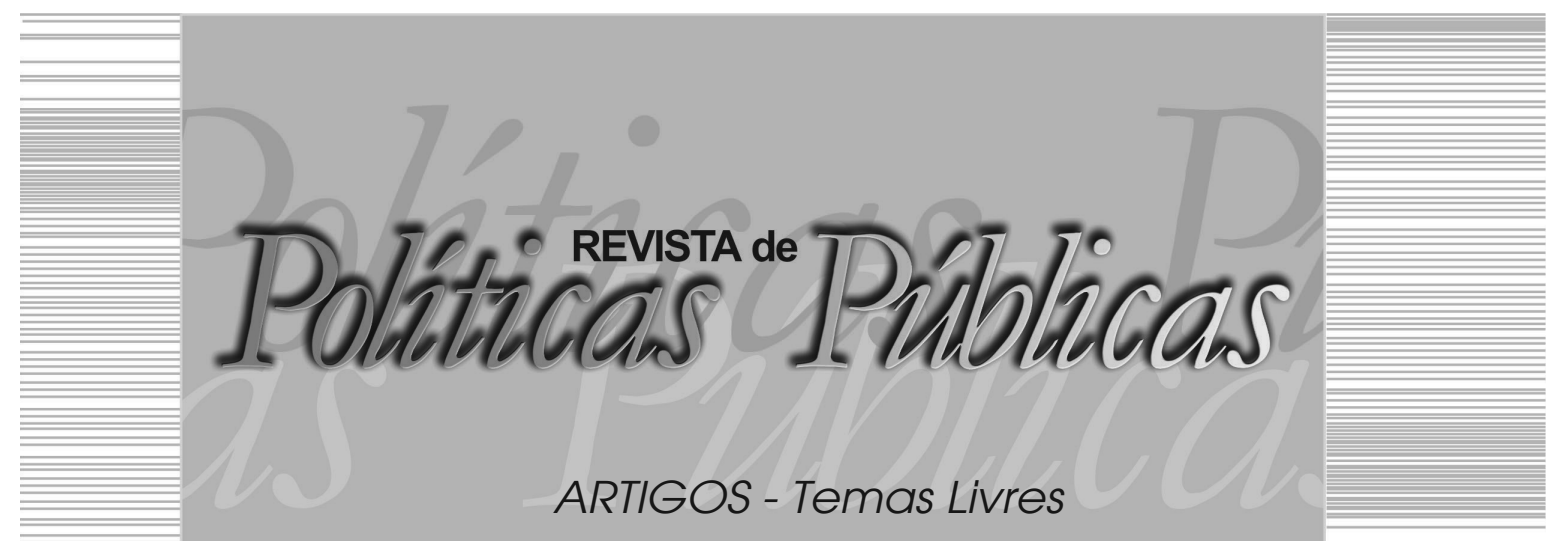

\title{
A INSTITUICIONALIZAÇÃO DA POLITICA DE SAÚDE MENTAL NO PARAGUAI: antecedentes, conteúdos e desafios atuais
}

\author{
Juliana Domingues ${ }^{1}$ \\ Universidade da Integração Latino Americana (UNILA) \\ João Alberto Gonçalves Junior ${ }^{2}$ \\ Universidade Católica de Pelotas (UCPel) \\ Vera Maria Ribeiro Nogueira ${ }^{3}$ \\ Universidade Federal de Santa Catarina (UFSC) \\ Universidade Católica de Pelotas (UCPel)
}

\section{Resumo}

As temáticas voltadas para a atenção em saúde mental no âmbito da América Latina vêm ganhando destaque na produção de conhecimento em face da ampliação no número de indivíduos com transtorno psíquico nesse espaço territorial, e a ausência, insuficiência e precariedade no atendimento. Ante essa situação, este artigo tem por objetivo apresentar a Política de Saúde Mental do Paraguai, regulamentada no ano de 2011, resgatando os antecedentes his-

\footnotetext{
Assistente Social, Doutoranda do Programa de Pós-Graduação em Políticas Sociais da Universidade Católica de Pelotas (UCPel), Professora da Universidade da Integração Latino Americana (UNILA). E-mail: judomingues16@gmail.com / Universidade da Integração Latino Americana - UNILA: Avenida Silvio Américo Sasdelli, 1842 - Vila A, Edifício Comercial Lorivo - Foz do Iguaçu - Paraná. CEP: 85866-000

2 Administrador, Mestre em Políticas Sociais pelo Programa de Pós-Graduação em Políticas Sociais da UCPe 1 Professor da UCPel. E-mail: joaoalbertojr7@gmail.com

3 Assistente Social, Doutora em Enfermagem pela Universidade Federal de Santa Catarina (UFSC), Professora Colaboradora da UFSC e Professora Adjunta da UCPel. E-mail: veramrn@gmail.com / Universidade Federal de Santa Catarina - UFSC: Campus Universitário Reitor João David Ferreira Lima, Bairro Trindade - Florianópolis - SC. CEP: 88040-900; Universidade Católica de Pelotas - UCPel: Rua Gonçalves Chaves, 373 , Sala 305B, Centro - Pelotas - RS. CEP: 96015-560
} 
tóricos, seus objetivos, ações programáticas e os principais desafios para sua efetivação. Assim, apresenta a relação entre a atenção à saúde mental, Direitos Humanos e o papel das organizações internacionais no caso paraguaio, a partir da realização de uma ampla pesquisa sobre o tema, em especial nos bancos de dados de organismos internacionais. Os achados do estudo evidenciam que o processo de implementação da Política de Saúde Mental venceu apenas um de seus muitos desafios: sua institucionalização no ano de 2011, sendo ainda necessária uma série de mudanças para seu efetivo propósito.

Palavras-chave: Política de Saúde Mental no Paraguai, reforma psiquiátrica, direitos humanos.

THE INSTITUTIONALIZATION OF MENTAL HEALTH

POLICY IN PARAGUAY: Current background, contests and challenges

\begin{abstract}
The issues surrounding the mental care in Latin America have been gaining a lot of attention in the production of knowledge duo the increase in the numbers of individuals with mental disorders in this territory and the absence, inadequacy and insecurity in the treatment. Given this situation, this article aims to present the Mental Health Policy of Paraguay, regulated in 2011, rescuing the historical background, its objectives, programmatic actions and the main challenges for its effectiveness. Thus it presents the relationship between mental health care, human rights and the role of international organizations in the Paraguayan case from conducting extensive research on the subject, especially in the databases of international organizations. The findings of the study show that the process of implementation of the Mental Health Policy has won only one of its many challenges: its institutionalization in 2011, still being needed a series of changes for its effective purpose.

Key words: Mental Health Policy in Paraguay, psychiatric reform, human rights.
\end{abstract}

\title{
1 INTRODUÇÃO
}

A produção de conhecimento sobre o diagnóstico e o desenvolvimento econômico e social na América Latina vem crescendo paulatinamente e evidencia, segundo o Informe Regional de Desarrollo Humano, avanços na redução da pobreza e desigualdade no crescimento econômico e na estabilidade financeira. (PROGRAMA DE LAS NACIONES UNIDAS PARA EL DESARROLLO, 2013.

$\mathrm{O}$ atual cenário, apesar de otimista, aponta ainda uma série de desafios a serem enfrentados, como as alarmantes estatísticas de 
violência e criminalidade; o uso abusivo de substâncias psicoativas, sobretudo entre as populações mais jovens; violência de gênero; crescente número de indivíduos acometidos por transtorno mental; baixos níveis de educação; corrupção; desemprego e subemprego; exploração do trabalho infantil e outras manifestações da questão social presentes nos países em desenvolvimento. (ORTIZ-HERNÁNDEZ; MORENO; BORGES, 2007).

Dentre os fenômenos mencionados, destaca-se a preocupante progressão dos transtornos psíquicos. Estes constituem um crescente e grave problema sanitário na América Latina e, segundo dados da publicação da Organización Panamericana de la Saúde (OPAS, 2007) sobre a Epidemiologia de Los Transtornos Mentales en América Latina y Caribe, em um período de dez anos, o número de pessoas acometidas por essas doenças aumentou de $12,5 \%$ para $14 \%$, sendo que uma a cada quatro pessoas no decorrer de sua vida desenvolverá algum tipo de doença mental. Poderá ainda se tornar a segunda maior causa de afastamento de trabalho.

A vulnerabilidade para o desenvolvimento de transtorno psíquico está fortemente associada às condições de vida, necessidades e iniquidades de saúde da população. Nos países da América Latina, essa vulnerabilidade se expressa na extrema pobreza, nas precárias condições de vida, trabalho e saúde da maioria da população que vive nesse espaço territorial, como a Comissão Econômica para a América Latina e Caribe (CEPAL, 2006) vem alertando nos seus relatórios. Essa situação é ainda potencializada pela ausência ou insuficiência de serviços públicos para atender às demandas, gerando um contingente de indivíduos completamente descobertos de atenção em saúde, em todos os níveis e especialidades de atendimento.

\begin{abstract}
Para poder hacerle frente a la creciente carga sanitaria que representan las enfermedades mentales en América Latina y el Caribe es menester entender su prevalencia y la brecha o hiato que existe en la provisión de atención, es decir, el porcentaje de personas que padecen de una afección determinado pero que no reciben ninguna atención [...] La carga de enfermedades mentales de la población no es solamente un reflejo de la prevalencia de estados psicopatológicos, sino el resultado de un conjuntos de estados e problemas sociales en los cuales el comportamiento desempeña papel preponderante. (KOHN et al., 2005, p. 10).
\end{abstract}

A situação identificada coloca-se como um desafio aos países da América Latina, especialmente ao Paraguai, evidenciando a 
necessidade da criação de serviços de caráter universal que incluam ações de promoção, prevenção, atenção e reabilitação das pessoas com transtorno psíquico e seus familiares. Observa-se que, em 2003, o país sofreu sanções da Comissão Interamericana de Direitos Humanos (CIDH) devido à situação degradante a que estavam sendo expostos os pacientes com transtorno psíquico no único hospital psiquiátrico do país. (ORGANIZACIÓN PANAMERICANA DE LA SALUD, 2006).

Esse cenário vem impondo questionamentos, tensionamentos e debates no país, apontando a imprescindibilidade na implementação de serviços voltados para o atendimento em saúde mental, previstos na legislação regulamentada em 2011, que institui a Política Nacional de Saúde Mental do Paraguai.

A precariedade das situações envolvendo o atendimento em saúde mental exigiu das autoridades sanitárias a inclusão do tema na agenda nacional do Paraguai e justifica a proposta desse estudo à medida que se busca evidenciar as formas de estruturação do Estado paraguaio e a conformação do sistema nacional de saúde e da Política Nacional de Saúde Mental. Esses aspectos incidem diretamente sobre as concepções e tendências de organização do Estado e das políticas sociais, interferindo substancialmente nos modelos de atenção e nas formas de garantia e acesso aos serviços de atenção à saúde mental.

A temática desse estudo deriva do Projeto de Tese da autora no Programa de Pós- Graduação em Política Social da Universidade Católica de Pelotas (UCPel) e se propõe a apresentar os antecedentes da implementação da Política de Saúde Mental no Paraguai e seus desafios de efetivação, evidenciando os aspectos conjunturais desse país, que ainda transita rumo à efetivação de um Estado democrático de direito que garanta a introdução dos princípios de defesa dos Direitos Humanos e da reforma psiquiátrica na construção da política de saúde mental.

Destaca-se ainda que, a crescente produção de conhecimentos sobre a atenção em saúde mental e a referência de boas práticas no Brasil, levou o governo paraguaio, no ano de 2007, a firmar um acordo de cooperação técnica com o Ministério da Saúde (MS) do Brasil, objetivando melhorar as condições da atenção em saúde mental no país. Tendo como modelo as diretrizes da Política de Saúde Mental brasileira, a interlocução entre os países objetivou ampliar 
e melhorar a capacidade de resposta do Estado mediante a criação de serviços comunitários para o tratamento de transtornos psíquicos. Esses aportes serviriam de modelo na construção da Política de Saúde Mental no Paraguai, instituída no ano de 2011. (BRASIL, 2011).

A maior parte dos dados que fundamentam esse estudo deriva de consultas realizadas em relatórios e pareceres de organismos internacionais (Organização Mundial da Saúde - OMS; OPAS; CEPAL; entre outras). Constatou-se que há um escasso número de publicações que tratem da atenção em saúde mental no Paraguai, principalmente no que tange a seu percurso histórico.

\section{PARAGUAI: uma terra cercada por terra}

No imaginário popular contemporâneo, pensar no Paraguai remete a duas perspectivas; a primeira, quanto a sua localização, compondo a tríplice fronteira com Brasil e Argentina, promovendo-o como parte integrante do roteiro turístico e de compras devido às Cataratas do Iguaçu e ao intenso comércio de produtos de naturezas diversas (eletrônicos, cosméticos, decoração, entre outros) que ocorre em Ciudad Del Este; e a segunda, e também muito conhecida, sobre a imagem de um país caracterizado pela grande mobilidade de pessoas e mercadorias, pelo cultivo e tráfico de drogas e armas, corrupção, informalidade no trabalho, criminalidade, violência, pobreza extrema, exploração e abuso do trabalho infantil. (SOUCHAUD, 2011).

O país, povoado inicialmente por índios da tribo Guarani, foi colonizado por espanhóis no século XVI e tem sua história marcada por grandes acontecimentos que conformam na atualidade as características locais. Entre tais, podemos destacar a Guerra da Tríplice Aliança (1864-1870), ou Grande Guerra como nominam os paraguaios; as ditaduras presentes em diversos momentos históricos; a aliança com o Brasil para a construção da Usina Hidrelétrica de Itaipu (1960) e a implantação de uma política de promoção da imigração internacional europeia, ocorrida na metade do século XIX, criando uma nação culturalmente diversificada. (GOIRIS, 2000).

O Paraguai, segundo a Dirección General de Estadísticas, Encuestas y Censos (DGEEC) (PARAGUAI, 2014), tem sua população atual estimada em aproximadamente 6,6 milhões de habitantes e uma de suas características singulares é a ausência de faixas litorâneas em suas terras, já que, dos países da América Latina, somente 
a Bolívia e o Paraguai não possuem fronteira oceânica. Essa peculiaridade impulsionou o governo na tentativa de tomar territórios argentinos e brasileiros próximos ao mar para desenvolver atividades comerciais e conquistar sua independência financeira, promovendo assim a Guerra do Paraguai.

Vários autores assinalam que as causas fundamentais da guerra tinham um fundo econômico-expansionista, no qual, a classe dominante de Buenos Aires, como também a nobreza do Império do Brasil, se prestavam para manter uma situação favorável à indústria britânica, provedora dos produtos manufaturados e importadora de matéria-prima. (GOIRIS, 2000, p. 16).

A guerra durou aproximadamente 10 anos e trouxe consequências terríveis para o país, sentidas ainda na contemporaneidade.

A guerra configurou-se numa forma de genocídio, cujas consequências econômicas, sociais e culturais, entre outras, repercutem até os dias de hoje. [...] de uma população de 550.000 mil habitantes, no início da guerra, em 1860, morreu mais da metade. Dos que restaram apenas 14.000 eram homens. Os sobreviventes eram mulheres, anciãos, crianças e mutilados. Além disso, o país perdeu 160 mil km² de seu território. A capital Assunção foi invadida e queimada, e Solano López refugiou-se na selva com o que sobrara de seu exército. (GOIRIS, 2000, p. 18).

Terminada a Guerra do Paraguai, iniciou-se um lento processo de reconstrução do país tendo como marco a promulgação da Constituição Nacional de 25 de novembro de 1870 e a criação dos partidos Liberal e Colorado no mesmo ano. Mesmo com a conformação de um Estado democrático de direito, os governos continuaram autoritários, com forte influência ditatorial, podendo o Paraguai ainda ser considerado um país em transição entre o seu histórico legado militar e a tentativa de implantação da democracia. (BIRCH, 1993).

Atualmente o Paraguai concentra a segunda maior comunidade de brasileiros no exterior, chegando a totalizar $350 \mathrm{mil} \mathrm{ha-}$ bitantes no ano de 2004. O país configura-se como um destino de terceirização de mão de obra não qualificada de setores industriais e do comércio do Brasil. A proximidade territorial entre o Paraguai e cidades brasileiras facilita a exportação de serviços em todos os modos de prestação e o livre comércio de pessoas e mercadorias, favorecendo o fluxo de investimento brasileiro no país. (BRASIL, 2004). 
Segundo relatório do Banco Mundial (BM, 2012), o Paraguai teve, no ano de 2012, o terceiro maior crescimento econômico do mundo: $14,1 \%$. Sua atividade econômica é impulsionada pela exportação de carne e soja, sendo considerado o sétimo e o quarto maior exportador, respectivamente. Destaca-se também a importação de eletrônicos e diversos produtos da China, comercializados livremente no país, beneficiados pela legislação que permite a livre circulação de bens e de divisas, contrapondo-se a um momento em que barreiras comerciais afetam a circulação de bens e a movimentação financeira em outros países da América Latina. Embora o Paraguai venha fortalecendo sua economia de mercado, evidencia-se: "La contradictoria economía paraguaya, en la que conviven formalidad y informalidad, capitalizados y descapitalizados, globalizados y marginados, abundancia y escasez, derroche y inanición debe ser comprendida en su totalidad, en su desarrollo histórico y en su lógica e, actual." (VILLAGRA, 2012, p. 14).

Na mesma proporção que cresce a economia, acentuam-se as desigualdades sociais, consagrando o Paraguai, conforme relatório da CEPAL (2013), como o segundo país com maior desigualdade social da América Latina. "Os dados mais recentes disponíveis indicam que o quintil mais pobre (isto é, 20\% dos domicílios de menor renda) capta, em média, $5 \%$ da renda total, com participações que variam de menos de 4\% em Honduras, no Paraguai e na República Dominicana." (COMISSÃO ECONÔMICA PARA A AMÉRICA LATINA E O CARIBE, 2013, p. 213).

O Paraguai reconheceu a saúde como um direito constitucional em 1967, garantindo a proteção e a promoção em saúde. Impõe a obrigatoriedade pessoal do cuidado, sendo que a concepção de saúde adotada nessa Constituição ainda remete à doença como centralidade do processo de atenção sanitária. (PARAGUAY, 1967). O artigo que trata do direito à saúde foi revisado na Constituição de1992 e permanece inalterado até o presente momento:

Art. 68 El estado protegerá y promoverá la salud como un derecho fundamental de la persona y en interés de la comunidad. Nadie será privado de asistencia pública para prevenir o tratar enfermedades, pestes o plagas, y de socorro en los casos de catástrofes y de accidentes. Toda persona está obligada a someterse a las medidas sanitarias que establezca la ley, dentro del respecto a la dignidad humana. (PARAGUAY, 1992, p. 45). 
O Ministerio de Salud Pública y Bienestar Social (MSPBS) é o órgão governamental responsável pela gestão da política de saúde no país e, embora o discurso constitucional remeta à prioridade nas ações de prevenção e promoção em saúde, a maior parte dos recursos financeiros ainda é gasta nos serviços de assistência à população. (DULLAK et al., 2011).

Segundo Arnau e Pieranton (1995), o Paraguai vem promovendo a gradual descentralização dos serviços de saúde, que se compõe de diversas instituições heterogêneas (centros e postos de saúde, hospitais, universidades, organizações não-governamentais, Cruz Vermelha paraguaia e uma extensa oferta de serviços privados). Porém, ainda sofre com limitações técnicas e financeiras que acabam por direcionar serviços à população mais carente, sendo que um grande contingente de pessoas ainda precisa recorrer aos serviços privados de saúde para receber atendimento.

\begin{abstract}
A pesar del marco legal establecido en la Constitución Nacional y de las instituciones encargadas de proteger la salud y promover La calidad de La ciudadanía paraguaya, en la práctica la atención totalmente gratuita es inexistente. Esto se debe a múltiplos factores, desde geográficos y políticos. Esta carencia convierte al Paraguay en un país donde, aún en el siglo XXI, el 1,2\% de población muere por diarrea, el 0,9 por enfermedades nutricionales y anemia. Además, el 78,3\% de los paraguayos carece cualquier seguro de salud. (PARAGUAY, 2008, p. 32).
\end{abstract}

É nesse contexto de adversidades decorrentes do parco investimento, da dificuldade em acessar serviços, do alto índice de desigualdade social que se potencializa a alta incidência de moléstias ligadas às precárias condições de vida e higiene de grande parte da população. Esses fatores aliam-se ainda à incipiente oferta de serviços e à falta de capacitação dos profissionais de saúde. Nesse cenário de percalços, a atenção em saúde mental também encontra inúmeros desafios para se efetivar enquanto uma política que rompa com o modelo manicomial historicamente reproduzido nos países em desenvolvimento.

\title{
3 REFORMA PSIQUIÁTRICA, DIREITOS HUMANOS E POLÍTICA DE SAÚDE MENTAL NO PARAGUAI
}

Os relatos sobre a ocorrência de transtornos mentais na história são tão antigos quanto a própria humanidade e revelam práticas desumanas a que esses indivíduos foram submetidos em função da 
cotidiana violência e segregação através de exorcismo, privação de água e comida, queima nas fogueiras pela Inquisição, castigos, abusos e tratamentos dolorosos. Foram sendo, pouco a pouco, isolados do convívio social, encarcerados e institucionalizados em asilos, manicômios e hospitais psiquiátricos, passando, não raras vezes, $\mathrm{o}$ resto de suas vidas nesses locais.

Desde o fim da Segunda Guerra Mundial, em 1945, as instituições de atendimento a pessoas com transtornos mentais, bem como os sistemas normativos e assistenciais psiquiátricos, sofreram reformas para tornar mais humanizado não só o tratamento, mas também a concepção de doença mental como um sofrimento humano e social, dando início a novas formas de olhar e tratar as pessoas com transtorno mental:

$$
\begin{aligned}
& \text { [...] pensamento que nasce como resposta à desumanização na Eu- } \\
& \text { ropa durante a Segunda Guerra Mundial, no qual era reportado o } \\
& \text { problema do Homem não mais como entidade abstrata, definível } \\
& \text { em um sistema de categorias fechadas, mas como um sujeito objeto } \\
& \text { de um sofrimento social. (AMARANTE, 1994, p. 64). }
\end{aligned}
$$

Entre as reformas psiquiátricas iniciadas em muitos países, destaca-se a italiana, impulsionada nos anos sessenta pelo psiquiatra Franco Basaglia (1974), alcançando visibilidade mundial ao questionar o saber, controle e poder exercido pela psiquiatria. Afirma o autor que o hospital psiquiátrico é "[...] um lugar de custódia, onde a ideologia médica é um álibi para a legalização de uma violência, que nenhuma organização está destinada a controlar, já que a delegação feita ao psiquiatra é total." (BASAGLIA, 1974, p. 34).

Basaglia (1974) defendia o fim do modelo hospitalocêntrico e propunha, em substituição a este, a implantação da psiquiatria preventivo-comunitária com oferta de serviços substitutivos em âmbito local, com a inclusão de profissionais de outras áreas (assistentes sociais, enfermeiros, psicólogos e terapeutas ocupacionais), apelando para o fim do histórico de abusos sofridos pelos doentes mentais privados do convívio social nos manicômios e asilos, fadados a uma condição de vida e de saúde sem dignidade humana.

A partir da reforma psiquiátrica italiana, o tema saúde mental passou a ser incorporado como pauta de organismos internacionais na defesa de Direitos Humanos de pessoas com enfermidades mentais, instaurando diretrizes para o atendimento, destacando-se a Declaração dos Direitos dos Retardados Mentais, proferida pela 
Organização das Nações Unidas (ONU) em 1971; o conjunto de normativas denominado Princípios, Directrices y Garantias para La Protección de Personas Detenidas a Título de Padecer de Enfermedades Mentales, criado pela CIDH, expedido no ano de 1984; e a Resolução da ONU, em 1991, que aprovou a Declaração sobre a Proteção de Pessoas Acometidas de Transtorno Mental e a Melhoria da Assistência em Saúde Mental. (ORGANIZACIÓN PANAMERICANA DE LA SALUD, 2006).

Na América Latina, a avaliação realizada no fim dos anos oitenta pela OPAS, sobre os serviços de saúde mental, identificou diversos problemas na superação do modelo antimanicomial e no desrespeito aos Direitos Humanos, sendo necessária a convocação da Conferência Regional para a Reestruturação da Atenção Psiquiátrica na América Latina, ocorrida no ano de 1990 em Caracas. O debate gerou diversas propostas que foram organizadas na Declaração de Caracas e os países signatários, entre eles o Paraguai, que se comprometeu a:

Promover os direitos humanos dos usuários; realizar ações de reestruturação da assistência psiquiátrica com ênfase na atenção primária e permanência em seu meio comunitário; rever o papel do hospital psiquiátrico e garantir os direitos civis e a dignidade pessoal das pessoas com transtorno psíquico. (ORGANIZACIÓN MUNDIAL DE LA SALUD; ORGANIZAÇÃO PANAMERICANA DE SAÚDE, 1990).

Mesmo tendo o Paraguai assumido o compromisso de implementar os serviços de saúde mental em consonância com os princípios da Declaração de Caracas, a CIDH solicitou, em dezembro de 2003, intervenção em caráter de emergência para proteger a vida e a saúde de 460 pessoas que estavam internadas no único hospital psiquiátrico localizado na capital do país. (HILLMAN, 2005).

A Comissão, em seu diagnóstico, relatou precárias condições estruturais; negligência com a higiene e alimentação dos internos; reduzido número de recursos humanos; crianças, homens e mulheres coabitando no mesmo espaço físico sem respeito a sua intimidade ou privacidade; superlotação na capacidade de atendimento; falta de atenção médica, psiquiátrica e odontológica; ausência de diagnóstico dos pacientes e profissionais sem capacitação; além de perpetuar a manutenção do tratamento hospitalar em detrimento do ambulatorial. (KOHN et al., 2005). 
Desde dezembro de 2003, a Agência Internacional para los Derechos de Personas con Discapacidad Mental e o Centro de Justicia y Derecho Internacional, em parceria com a OPAS, se mobilizam para mudar o cenário da atenção em saúde mental no Paraguai, não somente em relação às pessoas recluídas no hospital, mas também na estruturação de serviços comunitários.

Segundo o Informe do Who-AIMS sobre el Sistema de Salud Mental en Paraguay, os Direitos Humanos dos pacientes são supervisionados em alguns serviços, porém não existe um órgão de fiscalização no país. (ORGANIZACIÓN MUNDIAL DE LA SALUD; ORGANIZAÇÃO PANAMERICANA DE SAÚDE, 2006). Destaca-se, ainda, que apenas uma pequena parcela dos trabalhadores em saúde mental recebe formação em Direitos Humanos. Verificou-se, também, que a falta de recursos humanos e financeiros é um grave dificultador na implementação da proposta de tratamento ambulatorial e comunitário, sendo a internação a alternativa ainda mais utilizada para o tratamento de transtornos psíquicos.

Tais constatações remetem à preocupação com a garantia dos Direitos Humanos que, do ponto de vista legal, já tem reconhecido um conjunto de normativas legais em âmbito internacional, além de ter estruturadas agências locais e multilaterais específicas para fiscalizar a defesa dos padecedores de sofrimento psíquico. Porém, o contexto evidenciado neste estudo evoca a necessidade de refletir sobre a sua real efetivação:

Hablamos de derechos y parece que tal reconocimiento jurídico ya ha solucionado todo el problema que suponen las situaciones de desigualdad o injusticia que las normas deben regular. Sólo debemos preocuparnos de las garantías judiciales de los mismos, obviando absolutamente que tras todo El edificio jurídico subyacen sistemas de valores y procesos de división del hacer humano que privilegian a unos grupos y subordinan a otros. (FLORES, 2007, p. 13).

A discussão sobre a garantia dos Direitos Humanos aos padecedores de transtorno psíquico está além das garantias jurídicas já existentes, por residir na constante luta por sua real efetivação em um contexto de forte desigualdade social, que contribuiu para a regressão dos direitos sociais nos países da América Latina, com grande interferência das agências multilaterais na economia dos países, especialmente no Paraguai, considerado um país em desenvol- 
vimento, com imensas dificuldades de ordem política, econômica e social. (HILLMAN, 2005).

\section{ATENÇÃO EM SAÚDE MENTAL NO PARAGUAI}

Os registros sobre a história da atenção em saúde mental no Paraguai são escassos e credita-se esse fato ao tipo de desenvolvimento socioeconômico e cultural do país, que viveu boa parte de sua história sob o regime militar, que censurava o acesso à informação. Os achados da pesquisa bibliográfica e documental denunciam um forte traço de centralização da oferta de serviços, voltada para o modelo manicomial, além de diversos problemas identificados e publicizados no Instrumento de Evaluación para Sistemas de Salud Mental:

Se comprobó que, como únicamente el $2 \%$ de los profesionales médicos se dedican a salud mental, existen solo treinta y cinco profesionales del área por cada 100.000 habitantes, siendo en su mayoría psicólogos $(28,9: 10.000)$ En cuanto a los psiquiatras, la distribución de uno para cada 100.000. Además, no existen ni auxiliares capacitados para atender pacientes psiquiátricos. Escasean los trabajadores sociales y los terapeutas ocupacionales por una limitada oferta académica. En muchos centros de atención, son artesanos o personas entrenadas en oficios quienes hacen a la vez de terapeutas ocupacionales. (ORGANIZACIÓN PANAMERICANA DE LA SALUD, 2006, p. 147).

No Paraguai, o investimento nas ações e serviços da política de saúde mental, corresponde a $1 \%$ do orçamento destinado ao MSPBS. Desse montante, $84 \%$ são destinados ao único hospital psiquiátrico, localizado em Assunção, com capacidade de 400 leitos, aumentada em 8\% entre os anos de 2003 a 2005 para atender à crescente demanda. (ORGANIZACIÓN MUNDIAL DE LA SALUD; ORGANIZAÇÃO PANAMERICANA DE SAÚDE, 2006). Esta se compõe, em sua maioria, de pacientes com esquizofrenia, mantidos em regime de longa internação, contrapondo-se à tendência internacional de ênfase na criação de serviços ambulatoriais e comunitários. A rede de atendimento em saúde mental no Paraguai atualmente compõe-se de:

Centro de Día para Niños y Niñas con Trastornos Generalizados del Desarrollo (Centro de Salud Nro. 3) (ubicado en Asunción). Centro El Puente, para rehabilitación, acompañamiento terapéutico y terapia ocupacional de pacientes con trastornos mentales y retra- 
so mental leve (ubicado en Asunción). Centro Arte Día, para rehabilitación psicosocial por medio de arteterapia, musicoterapia, danzaterapia y acompañamiento psicológico a pacientes con trastornos mentales en general (ubicado en Asunción) Centro de Día Refugio para el Alma, un espacio de esparcimiento y acompañamiento terapéutico de personas con trastornos mentales, y el único del país con inclusión de familiares y amigos en sus proyectos (ubicado en Yaguarón, Departamento de Paraguarí). Centro de Atención a la Juventud, un servicio de salud general para población adolescente que incluye proyectos de rehabilitación y reinserción para pacientes con trastornos mentales (ubicado en Asunción) La población infantojuvenil es la más desfavorecida, pues no existen centros de internación que ofrezcan alas específicas o atención psiquiátrica para niños y adolescentes. (MOUJAN, 2011, p. 174).

Exceto pelo centro dia, toda a rede de serviços de reabilitação com equipe multiprofissional situa-se na capital do país, o que deixa totalmente descoberta a atenção em saúde mental nas demais cidades. Existem ainda 22 unidades de saúde que ofertam serviços de consulta psiquiátrica no Paraguai, dos quais $8 \%$ dos atendimentos destinam-se a crianças e adolescentes. Os serviços tratam 263 pacientes por 100.000 habitantes, sendo que 53\% dos usuários são do sexo feminino, destacando-se que, entre as consultas realizadas, $40 \%$ são de pacientes com menos de vinte anos de idade. Com relação ao diagnóstico, a maior incidência é de esquizofrenia (21\%) seguida dos transtornos afetivos (20\%). (MOUJAN, 2011).

Após a intervenção da Comissão de Direitos Humanos, no ano de 2003, em função das condições de saúde dos pacientes internados no hospital psiquiátrico em Assunção, houve outra fiscalização em 2005, na qual se verificou a melhoria das condições estruturais. (MOUJAN, 2011). Contudo, o país ainda precisaria implementar a oferta de serviços comunitários, sendo concedido para tal feito um prazo de cinco anos, com a garantia de que o país institucionalizaria uma lei nacional de saúde mental a partir dos princípios da reforma psiquiátrica.

Como parte de la liberación de los internos y en respuesta a las medidas cautelares, en el año 2007, se inicia un plan de hogares substitutos, cuyo objetivo es la reinserción comunitaria, se bien continúan con una visión manicomial de la atención en salud mental. Son tres los hogares substitutos económicamente independientes de los cuales dispone el país, aunque los mismos se encuentran en situación crítica por falta de apoyo financiero, social y cultural. (LÓPEZ, 2011, p. 1). 
Entre os anos de 2007 e 2008, segundo Lopez (2011), não houve melhorias na rede de serviços de atenção à saúde mental e, novamente, a CIDH impôs medidas cautelares ao Paraguai para melhorar a situação dos internos do hospital psiquiátrico, como também referenciou a urgente necessidade de investimento na rede extra-hospitalar.

À mesma época, o Paraguai firmou com o Brasil o Acordo de Cooperação Técnica por um período de doze meses. O objetivo foi o de melhorar as condições da atenção em saúde mental, tendo como modelo as boas práticas desenvolvidas na Política de Saúde Mental brasileira, ampliando a capacidade de resposta do Estado mediante a criação de serviços comunitários para o tratamento de transtornos psíquicos. (BRASIL, 2011). Posterior à assinatura do acordo, não há registro de avaliação da parceria, nem informações sobre a continuidade da cooperação.

Em 2009, o MSPBS do Paraguai organizou encontros com familiares, amigos, trabalhadores e gestores de saúde mental em algumas cidades para a discussão de bases para a criação da Política Nacional de Saúde Mental. (PARAGUAY, 2011). Aprovada em 2011, está pautada nos princípios da reforma psiquiátrica italiana que pretende transformar o atendimento em saúde mental respeitando os direitos dos usuários, tendo como princípio o atendimento comunitário e o rompimento com o modelo hospitalocêntrico, dando ênfase à qualidade de vida com equidade.

\begin{abstract}
Que al mismo tempo las Políticas para la Calidad de Vida y Salud con Equidad habla de cero indiferencia a la falta de medios adecuados para desarrollar una vida digna y plena y de seguridad social para los discapacitados, a su falta de acceso a un empleo digno, falta de datos e informaciones sobre la realidad que viven las personas con discapacidad, de rehabilitación y desarrollo de la autonomía, de iniquidad em el acceso a los servicios públicos de salud, de respuestas inequitativas para los distintos tipos de discapacidad, cero se diferencia a las causas prevenibles de todas las discapacidades, inclusive las causas traumáticas, infecciosas, degenerativas o de salud mental, además de lo referente a la mala calidad de atención en salud, cero a los abusos y violaciones por su condición de vulnerabilidad. (PARAGUAY, 2011, p. 23).
\end{abstract}

Os compromissos assumidos e as ações propostas por meio da atual Política Nacional de Saúde Mental (2011-2020) apontam a possibilidade de superação do modelo hospitalocêntrico-manico- 
mial. Propõe uma abordagem comunitária de saúde mental, baseada no respeito aos Direitos Humanos, no tratamento humanizado e na participação para a construção do exercício de cidadania das pessoas com transtorno psíquico. Assenta-se em quatro diretrizes filosóficas: universalidade, equidade, integralidade e na gestão participativa, possibilitando os pacientes com transtorno psíquico, as organizações de representação e familiares o controle social no planejamento, na gestão e execução das ações em saúde mental. (PARAGUAY, 2011).

A assistência, através da gratuidade e acessibilidade aos serviços, é reforçada pelos princípios da política, bem como a ampliação de serviços comunitários e de gestão nos territórios é ressaltada como uma ação relevante e necessária. Destaca-se ainda que a Política enaltece o respeito aos Direitos Humanos e identifica a necessidade de uma campanha de mudança cultural quanto à representação social do paciente com transtorno psíquico, com vistas à superação do modelo manicomial.

Desmanicomializar la cultura significa incluir en el desarrollo de las políticas de salud mental un multiverso de voces que abarque la de los profesionales de salud, de la sociedad civil, fundaciones y organizaciones que trabajan en el área. Significa desmontar el discurso hegemónico, reduccionista y biológico para sustituirlo por otro de carácter participativo, incluyente de otras disciplinas científicas y saberes populares. Y significa también rescatar las buenas prácticas, lãs respuestas efectivas que las propias comunidades utilizan para resolver problemas relacionados a la salud mental. (PARAGUAY, 2011, p. 28).

A Política Nacional de Saúde Mental do Paraguai prevê uma Rede Integrada de Serviços de Saúde Mental (RISS), estruturada nos níveis de atenção primária, especializada e hospitalar, além de serviços complementares como espaços substitutivos de integração (Centros Comunitários de Saúde Mental); redes de apoio e diagnóstico, assistência farmacêutica, vigilância em saúde e serviços de informação, transporte e reabilitação que atuem de forma interdisciplinar na implementação das ações em saúde mental. (PARAGUAY, 2011).

Embora a Política Nacional de Saúde Mental do Paraguai tenha sido implantada em 2011, até o momento não se identificou na literatura consultada como vêm se concretizando as ações propostas, ou seja, não há registros de estudos que permitam fazer a aferição da atual situação da atenção em saúde mental no país. 


\section{CONCLUSÃO}

O estudo permite concluir que as ações e serviços em saúde mental no Paraguai apresentam um desenvolvimento histórico marcado pela segregação, manicomialização e desrespeito aos Direitos Humanos dos pacientes com transtorno psíquico, potencializados pela precária situação econômica do país e pela incipiente oferta de serviços de caráter universal e gratuito em saúde mental, incidindo em muitos desafios a serem superados na implantação de uma política com vistas à superação do modelo manicomial.

No entanto, os esforços que o país vem realizando para a reversão desse quadro, aprovando uma Política Nacional de Saúde Mental com diretrizes pautadas nos princípios da reforma psiquiátrica, com ênfase na qualidade de vida com equidade e respeito aos Direitos Humanos, sugerem uma motivação para a implantação de ações e serviços comunitários de caráter descentralizado, universal e gratuito, impensáveis antes da criação dessa política.

O Paraguai é um país em desenvolvimento, vivenciando uma transição democrática que caminha a passos lentos na melhoria de todo o seu frágil sistema de seguridade social. Nesse contexto, é possível concluir que a implantação da Política Nacional de Saúde Mental é um desafio, sendo necessário a incessante luta pela efetivação dos princípios da reforma psiquiátrica nas ações em saúde mental, destacando-se ainda que a implementação das ações propostas necessita de apoio internacional, mediante alianças para o financiamento das proposições supracitadas, bem como da troca de boas práticas entre os países da América Latina. Assim, busca-se superar os desafios postos e alavancar os avanços necessários para a implantação de uma política de saúde mental de qualidade que atenda às necessidades da população usuária.

\section{REFERÊNCIAS}

AMARANTE, P. Uma aventura no manicômio: a trajetória de Franco Basaglia. História, Ciências, Saúde Manguinhos, Rio de Janeiro, v. 1, n. 1, p. 61-77, jul./out. 1994. Disponível em:<http://dx.doi. org/10.1590/S0104-59701994000100006>. Acesso em: 23 jun. 2014.

ARNAU, A.; PIERANTON, C. R. Paraguai: situação da formação e mercado de trabalho na área da saúde. In: ORGANIZAÇÃO PAN-AMERICANADA SAÚDE. Recursos humanos em saúde no 
Mercosul. Rio de Janeiro: FIOCRUZ, 1995. Disponível em: <http:// books.scielo.org/id/wcdsj/pdf/organizacao-9788575413982-06.pdf>. Acesso em: 2 maio 2014.

BANCO MUNDIAL. Relatório Anual de 2012. Washington, D.C., 2012. Disponível em: <http://web.worldbank.org/WBSITE/ EXTERNAL/EXTABOUTUS/EXTANNREP/EXTANNREP2012/0,,c ontentMDK:23278367 menuPK:8833388 pagePK:64168445 piPK:6 4168309 theSitePK:8784409,00.html>. Acesso em: 28 jun. 2014.

BASAGLIA, F. O homem do Pelourinho. Petrópolis, RJ: Vozes, 1974.

BIRCH, M. H. El legado económico en los años de Stroessner y el desafío por la democracia. In: BRUN, D. A. (Ed.). Paraguay en transición. Asunción: CADEP, 1993. Disponível em:<http://web. isanet.org/Web/Conferences/FLACSO-ISA\%20BuenosAires\%20 2014/Archive/9e94d2a8-7194-4840-83e4-48b5be283652.pdf $>$. Acesso em: 18 jun. 2014.

BRASIL. Ministério da Saúde. Secretaria de Atenção à Saúde. Departamento de Ações Programáticas Estratégicas. CoordenaçãoGeral de Saúde. Saúde Mental no SUS: as novas fronteiras da Reforma Psiquiátrica - Relatório de Gestão 2007-2010. Brasília, DF, 2011. Disponível em: $<$ http://docslide.com.br/documents/saudemental-no-sus-as-novas-fronteiras-dareforma-psiquiatrica-ministerio. html>. Acesso em: 1 jun 2016.

Ministério do Desenvolvimento, Indústria e Comércio Exterior. Secretaria de Comércio e Serviços. Oportunidades de negócios em serviços no Brasil e Paraguai. Brasília, DF, 2004.

COMISSÃO ECONÔMICA PARA A AMÉRICA LATINA E O CARIBE. Estudio Econômico del America Latina y el Caribe 20052006. Santiago de Chile, 2006. Disponível em: <http://www.cepal.org/ publicaciones/xml/5/26135/lcg2314e.pdf $>$. Acesso em: 2 jul. 2014.

Estudio Econômico del America Latina y el Caribe 2013. Santiago de Chile, 2013. Disponível em: http://repositorio.cepal.org/ bitstream/handle/11362/1252/S2013870_pt.pdf? sequence=1. Acesso em: 20 jan. 2015.

DULLAK, R. et al. Atención Primaria em Salud em Paraguay: panorámica y perspectiva. Ciência e Saúde Coletiva, Rio de Janeiro, v. 16, v. 6, p. 2865-2875, 2011.

FLORES, J. H. La reinvención de los derechos humanos. Andalucia: Atrapasuenõs, 2007. 
GOIRIS, F. A. J. Autoritarismo e democracia no Paraguai contemporâneo. Curitiba: UFPR, 2000.

HILMAN, A. A. Los derechos humanos y la exclaustración: un triunfo para el continente americano. Revista Panamericana de Salud Pública, Washington, D.C., v. 18, n. 4-5, p. 374-379, oct./nov. 2005. Disponível em: $<$ http://www.scielosp.org/scielo.php?script=sci_arttext\&pid $=\mathrm{S} 1020-49892005000900018>$. Acesso em: 21 jun. 2014 .

KOHN, L. I. et al. Los transtornos mentales en America Latina y el Caribe: asunto prioritario para la salud pública. Revista Panamericana de Salud Pública, Washington, D.C., 18, n. 4-5, p. 229-400, 2005.

LÓPEZ, C. Médico critica la "manicomialización” de institutos psiquiátricos. Diario ABC Color, Asunción, jun. 2011. Disponível em: $<$ http://www.abc.com.py/edicion-impresa/locales/medico-criticala-manicomializacion-de-institutos-psiquiatricos-275932.html $>$. Acesso em: 1 ago. 2014.

MOUJAN, M. C. A. Presente y futuro de los servicios de salud mental en Paraguay. Eureka: Revista Científica de Psicologia, Asunción, v. 8, n. 2, p. 171-182, 2011. Disponível em: <http://www.psicoeureka.com. py/publicacion/8-2/articulo/10>. Acesso em: jul. 2014.

ORGANIZACIÓN MUNDIAL DE LA SALUD; ORGANIZAÇÃO PANAMERICANA DE SAÚDE. Declaração de Caracas. Caracas, Venezuela, 1990. Não paginado. Conferência Regional para a Reestruturação da Atenção Psiquiátrica na América Latina no Contexto dos Sistemas Locais de Saúde (SILOS).

;___. Informe WHO - AIMS sobre el sistema de Salud Mental en Paraguay. Asunción, 2006. Disponível em: <http://www. who.int/mental_health/evidence/paraguay_who_aims_report_spanish. pdf>. Acesso em: 1 jul. 2014.

ORGANIZACIÓN PANAMERICANA DE LA SALUD.

Epidemiologia de los trastornos mentales en America Latina y Caribe. Washington, D.C., 2007. (Publicación Científica y Técnica, n. 632). Disponível em: $<$ http://www.paho.org/COL/index.php?option=com content\&view $=$ article $\& i d=355$ : epidemiologia-de-los-trastornosmentales-en-america-latina-y-el-caribe $\&$ catid $=698 \&$ Itemid $=361>$. Acesso em: jun. 2014.

. Evaluación para Sistemas de Salud Mental. Washington, D.C., 2006. Disponível em: $<$ http://www2.paho.org/hq/index. php?option $=$ com_content $\&$ view $=$ article $\&$ id $=445 \% 3 \mathrm{~A} 2008$ - 
A INSTITUCIONALIZAÇÃO DA POLITICA DE SAUDE MENTAL NO PARAGUAI: antecedentes, conteúdos e desafíos atuais

who-aims-general-information \&catid=1169\%3Atechnicalocuments\&Itemid=41252\&lang=es>. Acesso em: 1 jul. 2014.

ORTIZ-HERNÁNDEZ, L.; MORENO, S. L.; BORGES, G.

Desigualdad socioeconómica y salud mental: revisión de la literatura latinoamericana. Cadernos de Saúde Pública, Rio de Janeiro, v. 23, n. 6, p. 1255-1272, jun. 2007. Disponível em: <http://www.scielo.br/ scielo.php?script=sci_arttext\&pid=S0102-311X2007000600002>. Acesso em: 18 jun. 2014.

PARAGUAY. Constitución de la República del Paraguay. Asunción, 1967. . Asunción, 1992.

Dirección General de Estatísticas Escuestas y Censos. Censo de población 2014. Asunción, 2014. Disponível em: <htpp://www. dgeec.gov.py>. Acesso em: 19 jul. 2014.

Ministerio de Salud Pública y Bienestar Social. Dirección de Salud Mental. Política Nacional de Salud Mental 2011-2020. Assunción: OPS, 2011.

Paraguay: resumen de la analices de situación y tendencias de salud. Asunción, 2008. Disponível em: $<$ http:www.paho.org $>$. Acesso em: jun. 2014.

PROGRAMA DE LAS NACIONES UNIDAS PARA EL

DESARROLLO. Informe Regional de Desarrollo Humano 20132014: seguridad ciudadana con rostro humano: diagnóstico y propuestas para la America Latina. Nueva York, 2013. Disponível em: $<$ http://www.latinamerica.undp.org/content/dam/rblac/img/IDH/ IDH-AL\%20Informe\%20completo.pdf>. Acesso em: 1 jun. 2014.

SOUCHAUD, S. A visão do Paraguai no Brasil. Revista Contexto Internacional, Rio de Janeiro, v. 33, n. 1, p. 131-153, jan./jun. 2011.

VILLAGRA, L. R. (Org.). Processo histórico de La economia paraguaya. Asunción: Secretaria Nacional da Cultura el Paraguay, 2012. 
